\title{
Discrete $\beta$-function of the $S U(3)$ gauge theory with 10 massless domain-wall fermions
}

\section{Ting-Wai Chiu*}

Physics Department, National Taiwan University, Taipei 10617, Taiwan

Center for Quantum Science and Engineering, National Taiwan University, Taipei 10617,

Taiwan

Physics Department, National Taiwan Normal University, Taipei 11677, Taiwan

Institute of Physics, Academia Sinica, Taipei 11529, Taiwan

E-mail: twchiuephys.ntu.edu.tw

I present the updated results of the discrete $\beta$-function of the $S U(3)$ gauge theory with $N_{f}=10$ massless optimal domain-wall fermions in the fundamental representation. The renormalized coupling is obtained by the finite-volume gradient flow scheme on $L^{4}$ lattices, for seven lattice sizes: $L / a=8,10,12,16,20,24,32$; and each with 12 different lattice spacings parametrized by $6 / g^{2}$. The discrete $\beta$-function is extrapolated to the continuum limit using four lattice pairs $(L, 2 L) / a=(8,16),(10,20),(12,24)$ and $(16,32)$. This provides stronger evidence of the infrared fixed point at $g_{c}^{2} \sim 7$, which was first reported in Ref. [四], based on the continuum extrapolation of the discrete $\beta$-function obtained with three lattice pairs.

34th annual International Symposium on Lattice Field Theory

24-30 July 2016

University of Southampton, $U K$

\footnotetext{
*Speaker.
} 


\section{Introduction}

In the weak-coupling perturbation theory (WCPT), the $\beta$-function of the non-abelian gauge theory with $N_{f}$ massless fermion can possess a non-trivial infrared fixed-point (IRFP), besides the ultraviolet fixed point (UVFP) at $g^{2}=0$, provided that $N_{f}$ is within a range (i.e., the conformal window), and these theories are infrared conformal [ [ $]$, [3]. The relevance of these infrared conformal theories to high energy phenomenology is the possibility that the Higgs scalar in the Standard Model (SM) might be a bound state of fermion-antifermion in a new non-abelian gauge theory with $N_{f}$ massless fermions just below the edge of the conformal window, as an approximate Nambu-Goldstone boson resulting from breaking the conformal symmetry. This is one of the basic motivations of studying the $\beta$-function and the conformal window of non-abelian gauge theory with $N_{f}$ massless fermions. For a recent review, see, e.g., Ref. [四] and references therein.

In general, the conformal window depends on the gauge group as well as the representation of the massless fermions. For $S U(3)$ gauge theory with $N_{f}$ massless fermions in the fundamental representation, WCPT to 2-loop order gives the (approximate) conformal window $8<N_{f} \leq 16$ []]. For $N_{f}=10$, its IRFP is around $g^{2} \sim 28$. Obviously, WCPT is not supposed to give a reliable answer at such strong coupling. This calls for nonperturabtive study of the infrared behavior of the running coupling of nonabelian gauge theory with $N_{f}$ massless fermions. This is a fundamental problem in quantum field theory, regardless of whether the Higgs scalar is a composite scalar arising from the breaking of the conformal symmetry or not.

Lattice gauge theory provides a viable framework for nonperturbative study of vector gauge theories. Since we are dealing with massless fermions, it is vital to use lattice fermions with exact chiral symmetry, i.e., domain-wall [ []] /overlap [G, 四] fermions, having exactly the same flavor symmetry as their counterparts in the continuum. In this paper, we focus on the $S U(3)$ lattice gauge theory with 10 massless domain-wall fermions (DWF) in the fundamental representation. To preserve the chiral symmetry maximally on a lattice with finite extension in the fifth dimension, we use the optimal DWF with $R_{5}$ symmetry [ $[8]$, which has the effective 4-dimensional lattice Dirac operator exactly equal to the "shifted" Zolotarev optimal rational approximation of the overlap operator, with the approximate sign function $S(H)$ satisfying the bound $0 \leq 1-S(\lambda) \leq 2 d_{Z}$ for $\lambda^{2} \in\left[\lambda_{\text {min }}^{2}, \lambda_{\text {max }}^{2}\right]$, where $d_{Z}$ is the maximum deviation $\left|1-\sqrt{x} R_{Z}(x)\right|_{\max }$ of the Zolotarev optimal rational polynomial $R_{Z}(x)$ of $1 / \sqrt{x}$ for $x \in\left[1, \lambda_{\max }^{2} / \lambda_{\min }^{2}\right]$, with degrees $(n-1, n)$ for $N_{s}=2 n$.

To obtain the renormalized coupling of gauge theory on a finite lattice with volume $L^{4}$, we use the finite-volume gradient flow scheme [Q], which is based on the idea of gradient flow [ए人, ए]] to evaluate the expectation value $t^{2}\langle E\rangle$, where $E$ is the energy density of the gauge field, and $t$ is the flow time. This amounts to solving the discretized form of the following equation

$$
\frac{d B_{\mu}}{d t}=D_{v} G_{v \mu}
$$

with the initial condition $\left.B_{\mu}\right|_{t=0}=A_{\mu}$, where $G_{v \mu}=\partial_{v} B_{\mu}-\partial_{\mu} B_{v}+\left[B_{v}, B_{\mu}\right]$, and $D_{v} G_{v \mu}=$ $\partial_{\nu} G_{v \mu}+\left[B_{v}, G_{v \mu}\right]$. As shown in Ref. [U]], the gradient flow is a process of averaging gauge field over a spherical region of root-mean-square radius $R_{r m s}=\sqrt{8 t}$. Moreover, since $t^{2}\langle E\rangle$ is proportional to the renormalized coupling, one can use $c=\sqrt{8 t} / L$ as a constant to define a renor- 
malization scheme on a finite lattice, and obtain

$$
g_{c}^{2}(L, a)=\frac{16 \pi^{2}}{3[1+\delta(c, a / L)]}\left\langle t^{2} E(t)\right\rangle, \quad E(t)=\frac{1}{2} F_{\mu v} F_{\mu v}(t),
$$

where $a$ is the lattice spacing depending on the bare coupling $g_{0}, E$ is the energy density, and the numerical factor on the RHS of $(\mathbb{L})$ is fixed such that $g_{c}^{2}(L, a)=g_{\overline{\mathrm{MS}}}^{2}$ to the leading order. Here the coefficient $\delta(c, a / L)$ includes the tree-level finite volume and finite lattice spacing corrections [12]]. In this paper, we use the Wilson flow, the Wilson action, and the clover observable, the so called WWC scheme, which is known to have very small tree-level cutoff effects [ㅁ]]. Moreover, we fix $c=\sqrt{8 t} / L=0.30$.

For any input value of $g^{2}(L, a)=g^{2}$, we compute the discrete $\beta$-function (at finite $a$ )

$$
\beta\left(s, a / L, g^{2}\right)=-\frac{g^{2}(s L, a)-g^{2}(L, a)}{\ln \left(s^{2}\right)},
$$

for all lattice pairs $(L, s L)$ with fixed $s$. Assuming the discretization error of $\beta\left(s, a / L, g^{2}\right)$ behaves as $O\left(a^{2}\right)$, one can extrapolate $\beta\left(s, a / L, g^{2}\right)$ to the continuum limit, i.e., $\lim _{a \rightarrow 0} \beta\left(s, a / L, g^{2}\right)=$ $\beta\left(s, g^{2}\right)$, the so-called step-scaling method [[13]. Moreover, if $\beta\left(s, g^{2}\right)$ is determined for several values of $s$, then it can be extrapolated to $s=1$,

$$
\lim _{s \rightarrow 1} \lim _{a \rightarrow 0} \beta\left(s, a / L, g^{2}\right)=\lim _{s \rightarrow 1} \beta\left(s, g^{2}\right)=\beta\left(g^{2}\right)=\frac{d g^{2}}{d \ln \mu^{2}},
$$

which corresponds to the conventional $\beta$-function in the continuum. If $\beta\left(g^{2}\right)$ has an IRFP, then $\beta\left(s, g^{2}\right)$ also has a corresponding IRFP, and vice versa. In this paper, we determine the discrete $\beta$ function (with $s=2$ ) of $S U(3)$ lattice gauge theory with 10 massless optimal domain-wall fermions in the fundamental representaion, using four lattice pairs $(L, 2 L) / a=(8,16),(10,20),(12,24)$ and $(16,32)$ for extrapolation to the continuum limit $a \rightarrow 0$.

\section{Simulations}

We perform the HMC simulations with the Wilson plaquette gauge action and the optimal domain-wall fermion action with $R_{5}$ symmetry [8, [4]] on the 5-dimensional lattice $L^{4} \times 16$, for lattice sizes $L / a=8,10,12,16,20,24,32$, each with 12 bare coulings $\left(g_{0}\right)$ parametrized by $\beta=$ $6 / g_{0}^{2}=15.0,12.0,10.0,9.0,8.0,7.5,7.0,6.8,6.7,6.6,6.5,6.45$. Thus we have a total of 84 gauge ensembles. The boundary conditions of the gauge field are periodic in all directions, while the boundary conditions of the pseudofermion fields are antiperiodic in all directions. The simulations are performed on Nvidia GPUs. All gauge ensembles (except the $24^{4}$ and $32^{4}$ lattices at strong couplings) are simulated in one single stream, with one GPU or two GPUs in one computing node. For each gauge ensemble, we generate 4000-10000 trajectories after thermalization, and sample one configuration every 5 trajectories, which yields 800-2000 configurations for measurements. The statistical error of $t^{2}\langle E\rangle$ is estimated by the jackknife method with a bin size of 10-15 configurations of which the statistical error saturates. For any gauge ensemble, the statistical error of $t^{2}\langle E\rangle$ is less than $0.5 \%$ for $c=\sqrt{8 t} / L=0.3$. The chiral symmetry breaking due to finite $N_{s}=16$ is measured with the formula of the residual mass in Ref. [ㄷ]], and the residual mass for any gauge ensemble in this work is less than $5 \times 10^{-5}$. 


\section{Results}

Fixing $c=\sqrt{8 t} / L=0.3$, we measure the renormalized coupling $g^{2}(L, a)$ according to (ㅁ. $)$ ). The statistical error of $t^{2}\langle E\rangle$ is estimated by the jackknife method with a bin size of 10-15 configurations of which the statistical error saturates. For each gauge ensemble, the statistical error of $g^{2}(L, a)$ is less than $0.5 \%$. In Fig. 四, we plot the data of $1 / g^{2}(L, a)$ versus $a / L$, for 12 different values of $\beta=6 / g_{0}^{2}$. Here the $y$-axis is in the common logarithm scale. The data points with the same $\beta$ are connected by a dotted line. For each $\beta, 1 / g^{2}(L, a)$ is a monotonic increasing function of $a / L$.

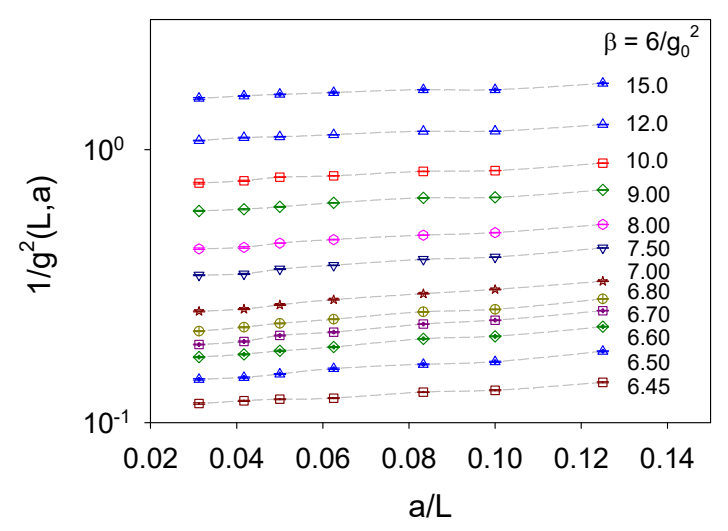

Figure 1: The data of $1 / g^{2}(L, a)$ versus $a / L$, for $L / a=8,10,12,16,20,24,32$, and for 12 values of $\beta=6 / g_{0}^{2}$ (as shown at the RHS of the figure). The data points of the same $\beta=6 / g_{0}^{2}$ are connected by a dotted line. The $y$-axis is in the common logarithm scale.

To compute the discrete $\beta$-function (ㄷ.2) for any $g^{2}(L, a)$, it requires $g^{2}(L, a)$ and $g^{2}(2 L, a)$ at the same $\beta=6 / g_{0}^{2}$ which in general is not equal to the 12 values of $\beta=6 / g_{0}^{2}$ in our simulations. In other words, the value of $g^{2}(L, a)$ at any $\beta \in[6.45,15.0]$ has to be obtained by interpolation, based on the 12 data points of $g^{2}(L, a)$ for each $L$. To this end, we use the cubic spline interpolation to obtain $g^{2}(L, a)$ for any $\beta \in[6.45,15.0]$. Then, for any input value of $g^{2}(L)$, the discrete $\beta$ function $(\mathbb{L} 2)$ can be obtained for any lattice pair $(L, 2 L)$. Since both the action and the observable only contain $O\left(a^{2}\right)$ corrections, the discrete $\beta$-function $\beta\left(2, a / L, g^{2}\right)$ can be linearly extrapolated to the continuum limit as a function of $(a / L)^{2}$, using 4 data points of different lattice spacings corresponding to the lattice pairs: $(8,16),(10,20),(12,24)$ and $(16,32)$.

In Fig. 凤, we plot $-\beta\left(2, a / L, g^{2}\right)$ versus $(a / L)^{2}$, for $g^{2}(L)=0.7,1.0,2.0,4.0,5.0,6.0,6.8$, and 7.0, together with the extrapolation to the continuum limit by the linear fit. For $g^{2}(L) \leq 6.80$, the data points are well fitted by a straight line with $\chi^{2} /$ d.o.f $<1$. At $g^{2}(L)=6.80$, the linear fit gives $-\beta\left(2, g^{2}\right)=0.09(6)$ with $\chi^{2} /$ d.o.f. $=0.71$, which is quite close to the IRFP. At $g^{2}(L)=6.90$, the linear fit gives $-\beta\left(2, g^{2}\right)=0.04(5)$ with $\chi^{2} /$ d.o.f. $=0.91$, which is closer to the IRFP than $g^{2}(L)=6.80$. At $g^{2}(L)=7.0$, the linear fit gives $\beta\left(2, g^{2}\right)=0.00(8)$ with $\chi^{2} /$ d.o.f. $=1.33$, which is consistent with zero up to the estimated error. This suggests that $g^{2}(L) \simeq 7.0$ is an IRFP of the discrete $\beta$-function of the $S U(3)$ gauge theory with $N_{f}=10$ massless fermions in the fundamental 

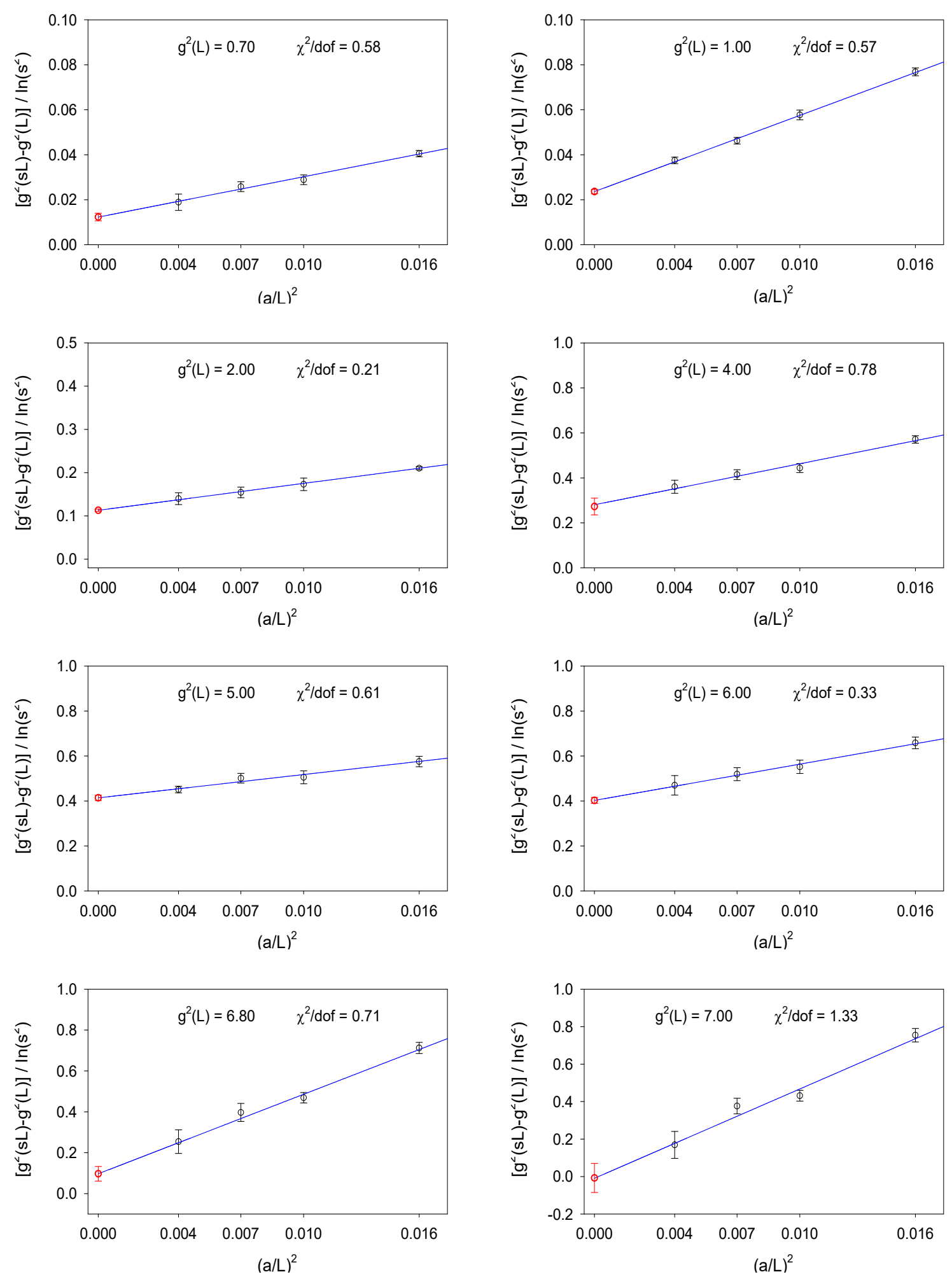

Figure 2: The discrete beta-functions of four lattice pairs $(L, s L) / a=\{(8,16),(10,20),(12,24),(16,32)\}$ are plotted versus $(a / L)^{2}$, for $g^{2}(L)=0.7,1.0,2.0,4.0,5.0,6.0,6.8$, and 7.0. The extrapolation to the continuum limit $(a \rightarrow 0)$ is obtained by linear fit, as shown by the straight line in each diagram. 
representation, in the finite-volume gradient flow scheme with $c=\sqrt{8 t} / L=0.3$.

The results of discrete $\beta$-function in the continuum limit $\beta\left(s, g^{2}\right)$ are plotted in Fig. [1], together with the 2-loop and 3-loop discrete $\beta$-functions in the $\overline{\mathrm{MS}}$ scheme.

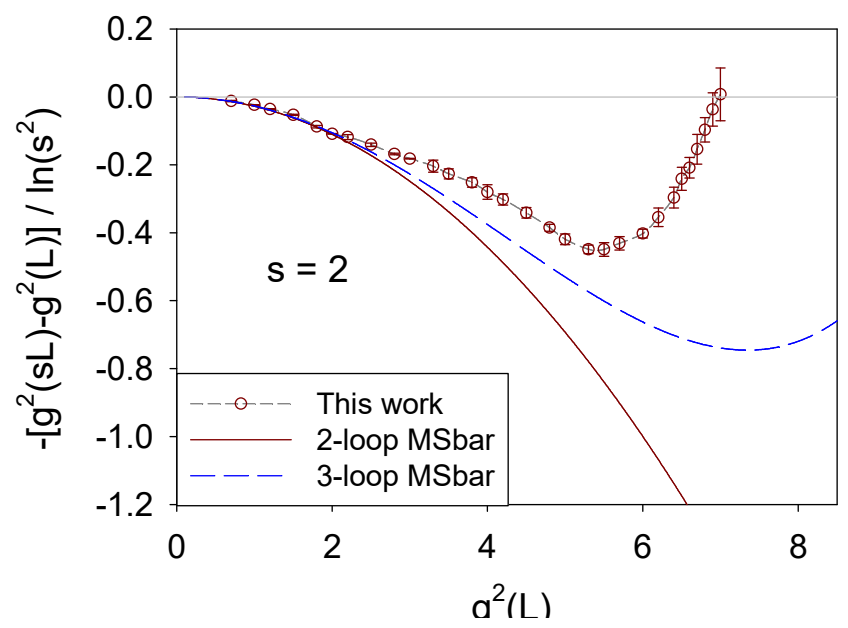

Figure 3: The discrete $\beta$-function in the continuum limit $\beta\left(2, g^{2}\right)$ versus $g^{2}$, for the $S U(3)$ lattice gauge theory with $N_{f}=10$ massless optimal DWF. The solid and dashed lines are the 2-loop and 3-loop discrete $\beta$-functions in the $\overline{\mathrm{MS}}$ scheme.

The salient features of the discrete $\beta$-functions in Fig. B can be summarized as follows. In the weak-coupling regime $g^{2}(L) \leq 2.2$, the lattice $\beta$-function is in good agreement with the 2-loop and 3-loop $\overline{\mathrm{MS}}$ results, as a decreasing function of $g^{2}(L)$. Then, in the regime $2.2 \leq g^{2}(L) \leq 5.3$, the lattice, the 2-loop, and the 3-loop $\beta$-functions are all decreasing functions of $g^{2}(L)$, but with different rates. In the strong-coupling regime, $5.3 \leq g^{2}(L) \leq 7.0$, the lattice $\beta$-function turns into an increasing function of $g^{2}(L)$ at $g^{2}(L) \simeq 5.3$, and it finally reaches IRFP at $g^{2}(L) \simeq 7.0$, while the 2-loop and 3-loop $\beta$-functions remain as decreasing functions of $g^{2}(L)$ with differnt rates. Note that the 3-loop $\beta$-function bends up to become an increasing function at $g^{2}(L) \simeq 7$.6, then attains its IRFP at $g^{2}(L) \simeq 10.6$ (out of the scale in Fig. B]).

\section{Concluding remarks}

In this work, the discrete $\beta$-function of $S U(3)$ lattice gauge theory with $N_{f}=10$ massless domain-wall fermions in the fundamental representation is determined with 84 gauge ensembles consisting of 7 lattice sizes $(L / a)^{4}=8^{4}, 10^{4}, 12^{4}, 16^{4}, 20^{4}, 24^{4}, 32^{4}$, and each lattice size of 12 values of $\beta=6 / g_{0}^{2} \in[6.45,15.0]$. The renormalized coupling is obtained by the finite-volume gradient flow scheme with $c=\sqrt{8 t} / L=0.3$, and the discrete $\beta$-function $\beta\left(2, a / L, g^{2}\right)$ is extrapolated to the continuum limit by the step-scaling method. The discrete $\beta$-function $\beta\left(2, g^{2}\right)$ in Fig. B shows that the theory possesses an infrared fixed point around $g^{2} \sim 7.0$, and it is infrared conformal. An estimate of the systematic errors due to: (i) the values of $g^{2}(L, a)$ are obtained by interpolation in $\beta=6 / g_{0}^{2}$, and (ii) the extrapolation of $\beta\left(s, a / L, g^{2}\right)$ to the continuum limit, will be presented in the updated version of Ref. [四]. 
Moreover, the interpolation error has been kept under control by simulating more gauge ensembles at some crucial values of $\beta=6 / g_{0}^{2}$, i.e, more than 12 values of $\beta$. To this end, I have introduced a criterion for adding a data point within the interval of two adjacent data points in $\beta$ such that the resulting interpolation error after adding this new data point can be reduced to one's desired accuracy. The details will be presented in the updated version of Ref. [四].

\section{Acknowledgments}

This work is supported by the Ministry of Science and Technology (Grant Nos. NSC1052112-M-002-016, NSC102-2112-M-002-019-MY3), Center for Quantum Science and Engineering (Grant Nos. NTU-ERP-103R891404, NTU-ERP-104R891404, NTU-ERP-105R891404), and National Center for High-Performance Computing. During the course of this work, I have benefitted from communications and discussions with many colleagues, in particular, Zoltan Fodor, Anna Hasenfratz, Julius Kuti, Herbert Neuberger, Claudio Pica, Benjamin Svetitsky, and Chik-Him Wong. I also thank Yu-Chih Chen, Han-Yi Chou, and Tung-Han Hsieh for their help in the code development. I am grateful to Zoltan Fodor and the Wuppertal Lattice Group for kind hospitality and support during my visit in the spring/summer of 2015.

\section{References}

[1] T. W. Chiu, arXiv:1603.08854 [hep-lat].

[2] W. E. Caswell, Phys. Rev. Lett. 33, 244 (1974).

[3] T. Banks and A. Zaks, Nucl. Phys. B 196, 189 (1982).

[4] C. Pica, PoS LATTICE 2016, 015 (2016) [arXiv:1701.07782 [hep-lat]].

[5] D. B. Kaplan, Phys. Lett. B 288, 342 (1992) [arXiv:hep-lat/9206013].

[6] H. Neuberger, Phys. Lett. B 417, 141 (1998) [hep-lat/9707022].

[7] R. Narayanan and H. Neuberger, Nucl. Phys. B 443, 305 (1995) [hep-th/9411108].

[8] T. W. Chiu, Phys. Lett. B 744, 95 (2015) [arXiv:1503.01750 [hep-lat]].

[9] Z. Fodor, K. Holland, J. Kuti, D. Nogradi and C. H. Wong, JHEP 1211, 007 (2012) [arXiv:1208.1051 [hep-lat]].

[10] R. Narayanan and H. Neuberger, JHEP 0603, 064 (2006) [hep-th/0601210].

[11] M. Luscher, JHEP 1008, 071 (2010) [JHEP 1403, 092 (2014)] [arXiv:1006.4518 [hep-lat]].

[12] Z. Fodor, K. Holland, J. Kuti, S. Mondal, D. Nogradi and C. H. Wong, JHEP 1409, 018 (2014) [arXiv:1406.0827 [hep-lat]].

[13] M. Luscher, P. Weisz and U. Wolff, Nucl. Phys. B 359, 221 (1991).

[14] Y. C. Chen and T. W. Chiu [TWQCD Collaboration], Phys. Lett. B 738, 55 (2014) [arXiv:1403.1683 [hep-lat]].

[15] Y. C. Chen and T. W. Chiu [TWQCD Collaboration], Phys. Rev. D 86, 094508 (2012) [arXiv:1205.6151 [hep-lat]]. 